\title{
Impacts des caractéristiques physico-chimiques des eaux sur la distribution du phytoplancton et des macrophytes de la rivière Nkam (Cameroun)
}

\author{
Victor Désiré TAFFOUO ${ }^{1 *}$, Rhode adley IKOLI SAYA ${ }^{2}$, Lawrence OBEN MBENG ${ }^{2}$ et \\ Minette TOMEDI EYANGO ${ }^{3}$
}

${ }^{I}$ Département de Biologie des Organismes Végétaux, Faculté des Sciences, B.P. 24157,

Université de Douala, Cameroun.

${ }^{2}$ Département d'Océanographie et de Limnologie, Institut des Sciences Halieutiques, B.P. 2701, Université de Douala, Cameroun.

${ }^{3}$ Département d'Aquaculture, Institut des Sciences Halieutiques, B.P. 2701, Université de Douala, Cameroun

*Auteur correspondant,E-mail :dtaffouo@yahoo.com

\section{RESUME}

Au Cameroun, les rivières sont soumises à des perturbations physiques et chimiques qui sont à l'origine d'une dégradation croissante de la qualité d'eau. La présente étude évalue l'impact des caractéristiques physico-chimiques des eaux sur la distribution et l'abondance du phytoplancton et des macrophytes de la rivière Nkam. Des inventaires floristiques ont été effectués à base des coefficients d'abondance-dominance de Braun-Blanquet et de sociabilité. Des mesures in-situ [ $(\mathrm{pH}$, température, oxygène dissous, conductivité électrique (COND) et solides totaux dissous (STD)] et des analyses chimiques [nitrates $\left(\mathrm{NO}_{3}{ }^{-}\right)$, phosphates $\left(\mathrm{PO}_{4}{ }^{2-}\right)$ et demande biochimique en oxygène $\left.\left(\mathrm{DBO}_{5}\right)\right]$ portant sur des échantillons d'eau prélevés dans les différentes stations de la rivière ont été effectuées dans le Laboratoire d'Analyses des Sols, Plantes, Engrais et Eau de l'Institut de la Recherche Agricole pour le Développement (IRAD), Yaoundé, Cameroun. Les macrophytes comptent 32 espèces distribuées dans 19 familles. La famille la plus diversifiée est celle des Poaceae avec six espèces (6) suivie des Asteraceae (5). Chez le phytoplancton, 80 espèces distribuées dans 58 genres et 14 familles ont été inventoriées. Les Diatomophyceae sont la plus diversifiée (25) suivie des Cyanobacteriaceae (18) et des Euglenophyceae (12). Les valeurs d'indice de diversité de Shannon Weaver traduisent une grande diversité spécifique du phytoplancton (4,09 à 4,54 bits) et des macrophytes $(2,28$ à 3,70 bits) de la rivière Nkam. La matrice de corrélation de Pearson montre que l'abondance des Cyanophyceae est corrélée positivement avec $\mathrm{PO}_{4}{ }^{2-}(\mathrm{r}=0,55)$, les Diatomophyceae avec $\mathrm{NO}_{3}{ }^{-}(\mathrm{r}=0,50)$, les Euglenophyceae avec COND $(\mathrm{r}=0,61)$ et la $\mathrm{DBO}_{5}(\mathrm{r}=0,77)$. Les Chlorophyceae, Bryopsidophycideae, Chlamydomonadaceae, Phacotaceae et Rivulariaceae ne sont positivement corrélées qu'avec le STD $(r=0,62)$. Les différentes espèces phytoplanctoniques et macrophytiques inventoriées donnent une indication sur la qualité de la rivière Nkam. Cette étude contribue à la gestion et la conservation des zones humides côtières.

(C) 2017 International Formulae Group. All rights reserved.

Mots clés : Inventaires floristiques, macrophytes, phytoplancton, caractéristiques physico-chimiques de l'eau, rivière Nkam-Yabassi. 


\title{
The impact of physico-chemical characteristics of water on the distribution of phytoplankton and macrophytes of Nkam River (Cameroon)
}

\begin{abstract}
In Cameroon, the rivers are subject to physical and chemical disturbances that are causing an increasing degradation of water quality. The present study was undertaken to assess the impact of the physico-chemical characteristics of water on the distribution and abundance of phytoplankton and macrophytes at the Nkam River. Floristic inventories were carried out on the basis of coefficient of abundance-dominance and sociability of Braun-Blanquet. The physical parameters [( $\mathrm{pH}$, temperature, dissolved oxygen, electrical conductivity (COND) and total dissolved solid (TDS) were measured in situ. The chemical parameters (nitrates $\left(\mathrm{NO}_{3}{ }^{-}\right)$, phosphates $\left(\mathrm{PO}_{4}{ }^{2-}\right)$, biochemical demand in oxygen (DBO5) were analyzed at the Soils, Plants, Fertilizers and Water Laboratory of the Institute of Agronomic Research for Development, Yaoundé, Cameroon. 32 species of macrophytes were found in 19 families. The most abundant family was Poaceae (6 species) followed by Asteraceae (5). An inventory of the phytoplanckton found a total of 80 taxa distributed in 58 genera and 14 families. The Diatomophyceae was the most abundant with 25 taxa followed by the Cyanobacteriaceae (18) and Euglenophyceae (12). The diversity index values of Shannon Weaver reflect a high specific diversity of phytoplankton (4.09 to 4.54 bits) and macrophytes ( 2.28 to 3.70 bits) of the Nkam River. Principal component analysis (PCA) showed a mineralization gradient downstream and the upload of the organic materials upstream. Pearson correlation matrix shows that Cyanophyceae is correlated positively with $\mathrm{PO}_{4}{ }^{2-}(\mathrm{r}=0.55)$, Diatomophyceae with $\mathrm{NO}_{3}{ }^{-}(\mathrm{r}=0.50)$, Euglenophyceae with COND $(\mathrm{r}=$ $0.61)$ and the DBO5 $(\mathrm{r}=0.77)$. The Chlorophyceae, Bryopsidophycideae, Chlamydomonadaceae, Phacotaceae and Rivulariaceae are correlated strongly with TDS $(r=0.62)$. The phytoplankton and macrophyte species may give an indication of the quality of the Nkam River contributing to the management and conservation of coastal wetlands.
\end{abstract}

(C) 2017 International Formulae Group. All rights reserved.

Keywords: Floristic inventories, macrophytes, phytoplancton, physico-chemical characteristics of water, Nkam River-Yabassi.

\section{INTRODUCTION}

L'accroissement démographique et l'essor de l'industrialisation sont des facteurs qui ont favorisé la pollution des écosystèmes aquatiques. La transformation de la société humaine a entrainé dans la nature des rejets constitués d'eaux résiduaires et de matières solides nuisibles à la vie et à la nature (Noukeu et al., 2016). Dans la plupart des pays africains les rivières sont soumises à des perturbations physiques et chimiques qui sont à l'origine d'une dégradation croissante de la qualité d'eau. Ces perturbations physicochimiques ont été mises en évidence au Togo lors d'une étude sur la caractérisation physicochimique et diversité phytoplanctonique des eaux du lac de Zowla (Atanle et al., 2012). En Côte-d'Ivoire ces perturbations sont observées dans quatre rivières (Soumié, Eholié, Ehania et Noé) du Sud-Est (Niamien-Ebrottié et al.,
2013) et dans le lac M'koa de Jacqueville (Kpidi et al., 2017). Au Sénégal les études sur le contrôle environnemental de la production primaire et les paramètres physico-chimiques et métalliques des eaux usées du canal Est qui débouche sur la baie de Hann ont été abordés (Sane, 2006; Diagne et al., 2017) tandis qu'au Cameroun, les études récentes ont été menées dans la ville de Yaoundé sur le Lac municipal (Kemka et al., 2004) et à Douala sur les rivières Kambo et Kondi (Priso et al., 2012 ; Dibong et al., 2014). Or, le Cameroun par son réseau hydrographique dispose d'un nombre important d'écosystèmes d'eau douce dont la qualité ne cesse de se dégrader. L'accroissement démographique, l'essor de l'industrialisation et la transformation de la société humaine relative au développement de l'agriculture qui utilise de plus en plus des produits phytosanitaires (engrais et pesticides) 
menacent l'équilibre des écosystèmes fluviaux et des communautés animales et végétales (Priso et al., 2010 ; Noukeu et al., 2016). Conséquemment, une intensification de l'eutrophisation avec des effets indésirables tels qu'une diminution de la biodiversité et un accroissement des espèces tolérantes à la pollution est observée (Roxane et Reinhard, 2015).

La pollution en milieu urbain peut être déterminée soit par des méthodes satellitaires, l'analyse des polluants ou des communautés végétales (Priso et al., 2010). Toutefois, nonobstant la pertinence des méthodes analytiques, elles restent de peu d'intérêts dans la détection des pollutions épisodiques (Ramade, 2008). La surveillance de la qualité de l'eau a longtemps été basée sur les analyses physico-chimiques afin de mettre en évidence des pollutions (Buchez et al., 2010). Par contre, les plantes intégratrices des phénomènes que connaissent les écosystèmes apparaissent comme des marqueurs relativement fiables des changements environnementaux (Sauberer et al., 2004). L'analyse biologique permet d'identifier les perturbations et leurs effets sur les communautés animales et végétales en place. En effet, dans des conditions de stabilité et d'enrichissement du milieu en substances nutritives favorables, une population d'algues peut se développer et selon les cas être bénéfique ou préjudiciable pour l'Homme (Evangelista et al., 2007; Reichwaldt and Ghadoua, 2012). En outre, plusieurs indices basés sur la sensibilité ou la tolérance à la pollution de certaines espèces d'algues microscopiques (Bacillariophyceae) ont été mis au point et utilisés en routine pour la surveillance biologique des réseaux hydrographiques (Bellinger and Sigee, 2015). L'hypothèse suivante sera vérifiée: les caractéristiques physico-chimiques de la rivière Nkam influencent la distribution et l'abondance des macrophytes et du phytoplancton.

L'objectif de cette étude est d'évaluer l'influence des paramètres physico-chimiques de l'eau sur la distribution et l'abondance des macrophytes et du phytoplancton, vérifier s'il existe des corrélations entre la flore aquatique et les paramètres physico-chimiques de l'eau et ressortir la distribution des macrophytes et $\mathrm{du}$ phytoplancton en fonction des caractéristiques physico-chimiques.

\section{MATERIEL ET METHODES}

Site d'étude et échantillonnage

Dans le cadre de la gestion et de la conservation des zones humides côtières, le site d'étude a été choisi dans l'arrondissement de Yabassi, Département du Nkam, Région du Littoral-Cameroun située entre $9^{\circ} 50^{\prime}$ et $10^{\circ} 10^{\prime}$ de latitude Nord; $4^{\circ} 20^{\prime}$ et $4^{\circ} 40^{\prime}$ de longitude Est, altitude : $15-20 \quad \mathrm{~m}$ (MINADER, 2011) (Figure 1). Le climat est équatorial de type guinéen et comporte quatre saisons (petite saison de pluies, petite saison sèche, grande saison de pluies et grande saison sèche) avec une pluviométrie moyenne de $1600 \mathrm{~mm}$, et une température moyenne annuelle de $25{ }^{\circ} \mathrm{C}$. Les sols sont de type ferralitique, les couches profondes $(>20 \mathrm{~cm})$ près des cours d'eau sont argileuses (MINADER, 2011). Trois stations ont été retenues en fonction du gradient longitudinal de la rivière Nkam.

La station 1 (N04²7'26.5" E009 $\left.{ }^{\circ} 8^{\prime} 33.3^{\prime \prime}\right)$ est située à Titina. La station 2 (N04²7'02.1" E00957'53.1") est située à 1,2 $\mathrm{km}$ de la station 1 et se trouve au marché de Yabassi. La Station 3 (N04²3'59.8" E009 $\left.52^{\prime 2} 27.9^{\prime \prime}\right)$ est située à Bodiman (Figure 1). Trois transects linéaires de 30,55 et $110 \mathrm{~m}$ chacun ont été effectués à chaque station pour les inventaires floristiques selon le gradient longitudinal du cours d'eau. Des quadrats de $1 \mathrm{~m}^{2}$ distants de $5 \mathrm{~m}$ ont été délimités de part et d'autre de chaque transect pour les relevés floristiques (Dibong and Ndjouondo, 2014). Les inventaires des macrophytes ont été effectués dans les stations 1,2 et 3 (Figure 1). 70 relevés de $1 \mathrm{~m}^{2}$ ont été réalisés. L'abondance-dominance de chaque relevé a été appréciée suivant l'échelle de Braun- Blanquet (1964) et l'indice de Masens (1997): $+=$ recouvrement $<1 \%$ correspondant à un recouvrement moyen de 0,$5 ; 1=$ recouvrement 1 à $5 \%$ correspondant à un recouvrement moyen de $3 ; 2=$ recouvrement 5 
à $25 \%$ correspondant à un recouvrement moyen de $15 ; 3=$ recouvrement 25 à $50 \%$ correspondant à un recouvrement moyen de 37,$5 ; \quad 4=$ recouvrement 50 à $75 \%$ correspondant à un recouvrement moyen de 62,$5 ; 5=$ recouvrement 75 à $100 \%$ correspondant à un recouvrement moyen de 87,5. La sociabilité des espèces suivant l'échelle a été ensuite donnée: $5=$ peuplements denses; $4=$ petites colonies; $3=$ petits groupes; $2=$ groupes restreints; $1=$ isolé.

Les indices d'abondance-dominance permettent de calculer pour chaque espèce son recouvrement moyen (RMi) qui est le pourcentage d'occupation moyen de cette espèce dans un milieu donné: $\mathrm{RMi}=$ (Ri/Nombre total de relevés) $* 100$

Avec Ri : recouvrement de cette espèce dans le milieu

L'indice de présence $\mathrm{Pi}$ correspond au recouvrement moyen (RMi) de l'espèce $i$ sur le recouvrement total des individus (WRM) : $\quad \mathrm{Pi}=\mathrm{RMi} / \mathrm{WRM}$

L'indice de Shannon Weaver (H') et l'équitabilité de Piélou (EQ) ont été choisis pour quantifier la diversité floristique.

L'indice de Shannon-Weaver (H') est noté

$$
H^{\prime}=-\sum_{i=1}^{s} \frac{N_{i}}{N} * \log _{2}\left(\frac{N_{i}}{N}\right)
$$

$\mathrm{Ni}$ : Nombre d'individus d'une espèce donnée $\mathrm{i}$, i allant de 1 à s (nombre totale d'espèces). $N$ : Nombre d'individus total. $\log$ : logarithme décimal

\section{L'équitabilité de Piélou}

$$
E Q=\frac{H^{\prime}}{\log _{2} N_{0}}
$$

$N_{0}:$ nombre total d'espèces

$H^{\prime}$ : indice de Shannon Weaver

Le coefficient de similitude de Jaccard ( $\mathrm{Sjij}$ met l'accent sur la présence de deux espèces au même endroit:

$\mathrm{Sjij}=[\mathrm{a} /(\mathrm{a}+\mathrm{b}+\mathrm{c})]$ avec $0<\mathrm{Sjij}<1$

$\mathrm{a}$ : nombre d'espèces communes aux deux milieux $\mathrm{b}$ : nombre d'espèces présentes dans le milieu A et absentes dans le milieu B

$\mathrm{c}$ : nombre d'espèces présentes dans le milieu $\mathrm{B}$ et absentes dans le milieu A

Le prélèvement des échantillons d'eau a été réalisé en quatre campagnes (première, deuxième, troisième et quatrième semaine $\mathrm{du}$ mois de juin 2015) entre 8 heures et 9 heures au niveau des différentes stations pendant la petite saison des pluies. La profondeur de prélèvement en plein eau a été d'environ 20 cm. 120 échantillons d'eau ont été prélevés lors des différentes campagnes. Tous les prélèvements d'eau ont été effectués dans des flacons de $250 \mathrm{ml}$ en polyéthylène tandis que ceux destinés à l'identification des espèces du phytoplancton se sont effectués dans des piluliers de $50 \mathrm{ml}$. Après prélèvement, les échantillons d'eau ont été mis dans une glacière et conservés entre 2 et $5{ }^{\circ} \mathrm{C}$ (Berho, 2008) jusqu'au Laboratoire d'Analyses des Sols, Plantes, Engrais et Eau de l'Institut de la Recherche Agricole pour le Développement (IRAD) de Nkolbisson-Yaoundé pour des analyses chimiques. Les échantillons du phytoplancton ont été immédiatement fixées au lugol, gardés au frais et à l'obscurité jusqu'au Laboratoire de Biologie des Organismes Végétaux de la Faculté des Sciences de l'Université de Douala. Chaque échantillon a été laissé au repos pendant $24 \mathrm{~h}$ au minimum pour permettre la sédimentation. L'eau a été ensuite prélevée au-dessus du résidu par siphonage. La solution résiduelle concentrée en phytoplancton a été conservée dans le pilulier. Quelques gouttes de chaque échantillon ont été montées entre lame et lamelle puis observées à l'aide d'un microscope optique (Olympus, B séries). Pour chaque échantillon, au moins cinq lames ont été préparées afin de s'assurer de la reproductibilité des lames (Atanle et al., 2012). Les observations ont été faites suivant des transects verticaux, ainsi que le comptage cellulaire sur la lame de comptage

\section{Analyse des paramètres physico-chimiques de l'eau \\ Le $\mathrm{pH}$ et la température ont été mesurés in situ par le multiparamètre}


HANNA (HI 98127). La mesure de la saturation en oxygène dissous (\%) a été effectuée in situ à l'aide de l'oxymètre HANNA (HI 9146). La conductivité électrique et les solides totaux dissous ont été également mesurés in situ à l'aide d'un conductimètre HANNA (HI8733). La méthode de dosage des nitrates est celle du salicylate de sodium. La lecture s'est faite à l'aide d'un spectrophotomètre à $415 \mathrm{~nm}$ (Rodier, 2009; Priso et al., 2014). La méthode colorimétrique au molybdate d'ammonium est utilisée pour le dosage des phosphates. La lecture au spectrophotomètre est effectuée à $440 \mathrm{~nm}$ contre le réactif blanc (Rodier, 2009). La demande biologique en oxygène est obtenue par le piège à anhydride carbonique dans une solution concentrée de soude à l'aide d'une pompe doseuse (Rodier, 2009).

\section{Analyses des données}

Dans le cadre de cette étude, une Analyse en Composantes Principales (ACP) du programme de traitement statistique Xlstat a été réalisée avec l'ensemble des données recueillies au niveau des différents sites d'étude. Le Tableau de contingence comprend ainsi les échantillons définis chacun par sa composition spécifique et l'abondance des différents taxons de microphytes ou de macrophytes ainsi analysés. Les valeurs moyennes d'indice de diversité de Shannon Weaver ( $\left.H^{\prime}\right)$ dans les différents sites d'études ont été comparées en appliquant le test d'ANOVA. L'ANOVA a été complétée par le test de Games-Howell pour analyser la différence entre les valeurs moyennes de $\mathrm{H}^{\prime}$ obtenues dans les 3 stations. La matrice de corrélation de Pearson a permis de déterminer les coefficients de corrélation les plus significatifs entre les variables abiotiques et les variables biotiques. Le logiciel SPSS version 12.0 a été utilisé pour la réalisation de cette analyse statistique.

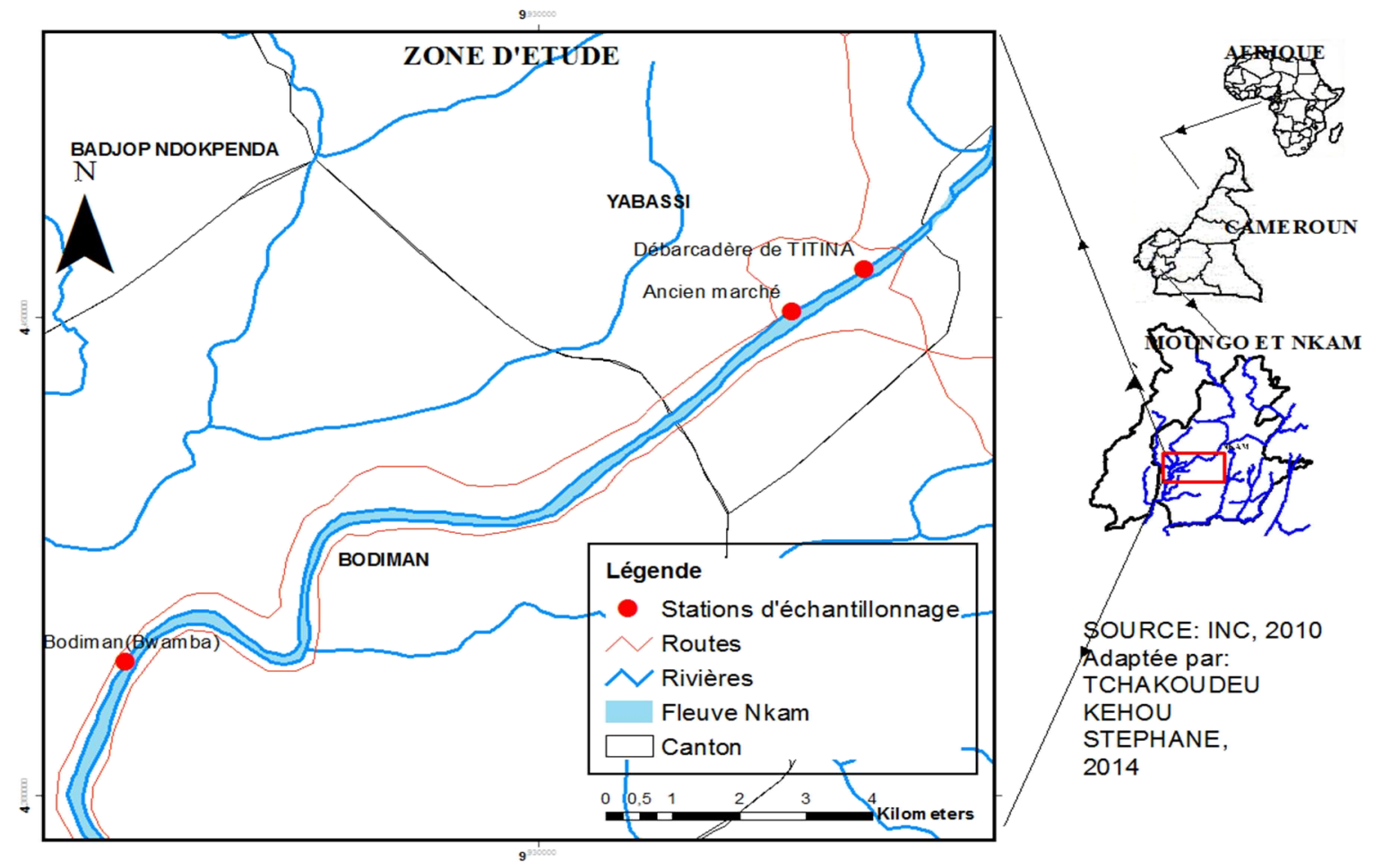

Figure 1: Carte d'échantillonnage représentant les différentes stations entre Titina et Bodiman dans la rivière Nkam. 
RESULTATS

Paramètres physico-chimiques des eaux des stations d'étude

La température ambiante (TA) est élevée dans la station 1 (amont) et 3 (aval) $\left(29,80^{\circ} \mathrm{C}\right)$. La conductivité électrique (COND) fluctue entre $213 \mu \mathrm{S} / \mathrm{cm}$ dans la station 1 et $217 \mu \mathrm{S} / \mathrm{cm}$ dans la station 2 (Tableau 1). Les valeurs en nitrates $\left(\mathrm{NO}_{3}^{-}\right)$sont de $0,46 \mathrm{mg} / \mathrm{len}$ amont et de $0,57 \mathrm{mg} / 1$ en aval. Le taux de phosphate $\left(\mathrm{PO}_{4}{ }^{2-}\right)$ est de $0,09 \mathrm{mg} / \mathrm{l}$ en amont et de $0,17 \mathrm{mg} / 1$ en aval. Le taux d'oxygène dissous $\left(\mathrm{O}_{2}\right)$ est de $4,22 \mathrm{mg} / \mathrm{l}$ en amont et de $3,54 \mathrm{mg} / 1$ en aval. Globalement, l' $\mathrm{O}_{2}$ dissous en amont est non déficitaire au seuil fixé à 4 $\mathrm{mg} / \mathrm{l}$ comparativement à l'aval. Les valeurs de $\mathrm{DBO}_{5}$ sont de $2,02 \mathrm{mg} / \mathrm{l}$ en amont et de 2,27 $\mathrm{mg} / \mathrm{l}$ en aval. Le taux de solides totaux dissous (STD) est de 185,58 ppm en amont et de 176,44 ppm en aval.

Les corrélations de Pearson montrent que les variables sont significativement corrélées deux à deux à $\mathrm{p}<0,05$. Les valeurs des coefficients de corrélation sont proches de 1 entre la TA et $\mathrm{PO}_{4}{ }^{2-}(\mathrm{r}=0,96, \mathrm{p}<0,01)$, TS et $\mathrm{PO}_{4}{ }^{2-}(\mathrm{r}=0,96, \mathrm{p}<0,01)$, COND et DBO5 $(\mathrm{r}=$ $0,95, p<0,05)$, COND et salinité $(r=0,88$, $\mathrm{p}<0,05)$. Des fortes corrélations négatives ont été observées entre COND et TA $(r=-0,93, p$ $<0,05)$, salinité et TA $(r=-0,98 \mathrm{p}<0,01)$, DBO5 et TA $(r=-0,98, p<0,05)$, TS et DBO5 $(\mathrm{r}=-0,92, \mathrm{p}<0,05)$, COND et $\mathrm{O}_{2}$ dissous $(\mathrm{r}=$ $-0,95, \mathrm{p}<0,05)$, salinité et $\mathrm{PO}_{4}{ }^{2-}(\mathrm{r}=-0,97, \mathrm{p}$ $<0,01), \mathrm{O}_{2}$ dissous et DBO5 $(\mathrm{r}=-0,94, \mathrm{p}<$ 0,05). Les plans factoriels F1x F2 de l'analyse en composantes principales (ACP) apportent $88,16 \%$ de l'ensemble d'informations (Figure 2). L'analyse de l'organisation des variables sur les plans factoriels F1 x F2 de 1'ACP a révélé que l'axe factoriel $\mathrm{F} 1$ oppose deux ensembles de variables. Il est corrélé positivement avec les éléments du premier ensemble contenant des descripteurs corrélés: COND $(r=0,92)$, STD $(r=0,95)$, DBO5 $(r=$ $0,79)$, salinité $(\mathrm{r}=0,78)$ et $\mathrm{NO}_{3}{ }^{-}(\mathrm{r}=0,66)$. Par contre, TA $(r=-0,84)$, TS $(r=-0,84)$ et $\mathrm{O}_{2}(r$ $=-0,85)$ sont corrélées négativement avec l'axe F1. L'axe F1 définit donc des éléments de minéralisation suivant le gradient amont-aval.
L'axe F2 est formé d'un ensemble des variables corrélées avec cet axe et qui s'opposent à l'ensemble des variables de l'axe F1. Ce sont: $\mathrm{PO}_{4}{ }^{2-}(\mathrm{r}=-0,61), \mathrm{pH}(\mathrm{r}=0,96)$ et $\mathrm{O}_{2}$ dissous $(\mathrm{r}=-0,85)$.

\section{Diversité du phytoplancton}

$\mathrm{La}$ richesse spécifique $\mathrm{du}$ phytoplancton des différents sites d'étude de la rivière Nkam est diversifiée et comporte 58 genres et 80 espèces distribuées dans 14 familles. La classe des Diatomophyceae est la plus diversifiée avec vingt-cinq (25) espèces, ensuite viennent les Cyanophyceae (18), les Euglenophyceae (12), les Chlorophyceae (8), les Xanthophyceae (4), les Zygophyceae (4), les Dinophyceae (2), les Bryopsidophycideae (1), les Chlamydomonadaceae (1), les Cryptophyceae (1), les Cyanobacteriaceae (1), les Phacotaceae (1), les Rivulariaceae (1) et les Ulothricophyciaceae (1).

Le Tableau 2 indique les valeurs moyennes de l'indice de diversité de Shannon Weaver et d'équitabilité de Pielou du phytoplancton dans les stations 1 et 3 . L'indice de diversité spécifique varie de 4,09 à 4,52. L'équitabilité de Pielou varie de 0,97 à 1,00 .

La Figure 3 présente la proportion d'algues (\%) en amont et en aval sur la rivière Nkam. Il ressort de cette figure que les Bacillariophyceae et Cyanophyceae représentent 15 et $7 \%$ en amont, 17 et $16 \%$ en aval, respectivement tandis que les Euglenophyceae et les Xanthophyceae comptent $4 \%$ chacun en amont et 7 et $5 \%$ en aval, respectivement. Cependant pour les Chlorophyceae la proportion est identique en amont et en aval (5\%).

\section{Corrélation entre les variables physico- chimiques et phytoplancton}

La projection des familles sur les axes F1 x F2 (70,95\%) avec des variables sur les mêmes axes, laissent entrevoir clairement que le site 3 (aval) coïnciderait avec le pôle minéral et serait alors soumis à une forte pollution minérale (Figure 4). Cependant le site 1 (amont) apparaît être le site où la 
présence de $\mathrm{PO}_{4}{ }^{2-}$ favoriserait la croissance des végétaux et $\mathrm{l}^{\prime} \mathrm{O}_{2}$ dissous une décomposition de la matière organique. La matrice de corrélation de Pearson révèle que parmi les différentes familles phytoplanctoniques, l'abondance des Cyanophyceae est corrélée significativement et positivement avec $\mathrm{PO}_{4}^{2-}(\mathrm{r}=0,55)$, TS $(\mathrm{r}=$ $0,73)$ et TA $(r=0,74)$ (Figure 4). Les Diatomophyceae et Bacillariophyceae avec le $\mathrm{pH} \quad(\mathrm{r}=0,56)$ et $\mathrm{NO}_{3}^{-}(\mathrm{r}=0,50)$, les Chlorophyceae avec STD $(r=0,62)$ et $\mathrm{NO}_{3}^{-}$ $(\mathrm{r}=0,42)$, les Euglenophyceae avec $\mathrm{pH}(\mathrm{r}=$ $0,87)$, COND $(r=0,61)$ et DBO5 $(r=0,77)$, les Xanthophyceae avec le $\mathrm{pH}(\mathrm{r}=0,56)$. Les Bryopsidophycidaceae Chlamydomonadaeae, Phacotaceae et Rivulariaceae ne sont positivement corrélés qu'avec le STD ( $\mathrm{r}=$ $0,62)$.

\section{Diversité des macrophytes}

La richesse spécifique des macrophytes de l'ensemble des sites d'étude est diversifiée et comporte 32 espèces distribuées dans 19 familles. La famille la plus diversifiée est celle des Poaceae avec six espèces (6), Asteraceae (5), puis les Amaranthaceae (2), les Commelinaceae (2), les Convolvulaceae (2), les Acanthaceae (1), les Apocynaceae (1), les Cesalpiniaceae (1), les Costaceae (1), les Cyperaceae (1), les Rubiaceae (1), les Malvaceae (1), les Onagraceae (1), les Mimosaceae (1), les Euphorbiaceae (1), les Rutaceae (1), les Solanaceae (1), et les Cucurbitaceae (1).

Les valeurs de l'indice de Shannon Weaver (H') et d'équitabilité de Pielou (E) des macrophytes dans les stations 1,2 et 3 sont représentées dans le Tableau 3. Il ressort de ce Tableau que chez les macrophytes les valeurs d'indice de diversité de Shannon Weaver varient de l'aval vers l'amont de 2,28 à 3,70 bits et l'équitabilité de Pielou de 0,79 à 0,88 . L'amont présente une diversité plus importante (22 espèces) que l'aval (10 espèces).

L'indice de recouvrement moyen montre que Struchium sparganophora est l'espèce la plus abondante dans la station 2 avec un recouvrement moyen (RM) de 30\% sur l'ensemble des relevés effectués dans la zone d'étude (Figure 5). Sa présence est observée dans toutes les stations étudiées. Vernonia amygdalina et Echinochloa pyramidalis sont les plus abondantes dans les stations 2 et 3 avec $10 \%$ RM. Toutefois, elles sont absentes dans la station 1. Diodia scandens est présente dans toutes les stations. Cependant, elle est plus abondante dans la station 1 avec $10 \%$ RM et moins abondante dans les stations 2 et 3 avec 5\% RM. Alstonia boonei et Mimosa pudica sont uniquement présentes dans la station 1 (5\% et moins de 5\% RM, respectivement). Costus afer et Eleusine indica sont présentes dans la station 2 uniquement avec moins de 5\% RM.

Le Tableau 4 indique les caractéristiques phyto-sociologiques des sites d'étude. Il ressort de ce Tableau que la station 1 présente une pente de $5 \%$, une largeur du lit mouillé de $100 \mathrm{~m}$ avec des habitats et des cultures le long des berges. La station 2 a une pente faible $(0,5 \%)$, une largeur du lit mouillé de $110 \mathrm{~m}$. Les habitats et les cultures se trouvent également le long des berges. Quant à la station 3, la pente est de $0,5 \%$, la largeur du lit mouillé est de $100 \mathrm{~m}$. Les habitats et les cultures (maïs, piment, orangers, palmiers, cacaoyers et papayers) longent la rivière Nkam.

\section{Groupements végétaux en relation avec le milieu}

Au total 70 relevés de $1 \mathrm{~m}^{2}$ ont été réalisés. La végétation $\mathrm{du}$ site étudié s'organise en groupements végétaux dans lesquels cohabitent des espèces qui y trouvent des conditions favorables. Les facteurs écologiques prépondérants ne sont pas les mêmes pour toutes les espèces réunies. Le groupement végétal résulte de la juxtaposition de groupes d'espèces liés chacun à la variation des facteurs écologiques. Les principaux groupements rencontrés sont:

- le groupement 1 (8 relevés) de la station 1 possède 77 espèces parmi lesquelles Ipomoea batatas, Echinochloa pyramidalis, Asystasia gangetica, Phyllanthus muelerianus, Alstonia boonei, Hibiscus 
esculentum et Ludwigia adscendens (Tableau 5). Ce groupe couvre une longueur de $16 \mathrm{~m}$. Ipomoea batatas et Echinochloa pyramidalis sont les espèces caractéristiques de ce groupe et apparaissent dans tous les 8 relevés. Asystasia gangetica apparaît comme une espèce compagne et se retrouve dans 4 relevés sur 8 .

- le groupement 2 (4 relevés) de la station 1 comporte 6 espèces: Dioda scandens, Chromolaena odorata, Solanum nigrum, Amaranthus viridus, Cassia obtusifolia et Panicum maxicum (Tableau 5). Ce groupe couvre une longueur de $8 \mathrm{~m}$. La moyenne d'espèces pour ce groupe est de 3,7. Diodia scandens est une espèce caractéristique de ce groupe et apparait dans 3 relevés sur 4. Chromolaena odorata apparait dans 2 relevés sur 4 et constitue une espèce compagne. Panicum maximum apparait comme accidentel.

- le groupement 3 (3 relevés) de la station 1 possède 5 espèces: Alternanthera sp., Pennisetum felicianum, Vernonia amygdalina, Setaria barbata et Leersia hexandra (Tableau 5). Ce groupe couvre une longueur de $6 \mathrm{~m}$. Pennisetum felicianum est une espèce caractéristique de ce groupe et apparait dans 3 relevés sur 3. Vernonia amygdalina et Setaria barbata sont des espèces compagnes. Elles apparaissent dans 2 relevés sur 3.

- le groupement 4 (2 relevés) de la station 1 comporte 4 espèces: Ipomoea purpurea, Momordica charantia, Struchium sparganophora et Ageratum conyzoides (Tableau 5). Ce groupe couvre une longueur de $4 \mathrm{~m}$. La moyenne d'espèces pour ce groupe est de 1. Les espèces de ce groupe sont des espèces compagnes et apparaissent dans 2 relevés sur 4.

- le groupement 5 (22 relevés) de la station 2 possède 4 espèces: Pennisetum felicianum, Momordica charantia, Ipomoea batatas, et Asystasia gangetica (Tableau 6). Ce groupe couvre une longueur de $44 \mathrm{~m}$. La moyenne d'espèces pour ce groupe est de 2,22. Pennisetum felicianum et Momordica charantia sont des espèces caractéristiques de ce groupe. Elles apparaissent dans 19 et 14 relevés sur 22 , respectivement.

- le groupement 6 (13 relevés) de la station 2 comporte 5 espèces: Amaranthus viridus, Setaria barbata, Struchium sparganophora, Chromolaena odorata et Cassia obtusifolia (Tableau 6). Ce groupe couvre une longueur de $26 \mathrm{~m}$. Les espèces de ce groupe sont considérées comme des espèces compagnes. Elles apparaissent dans 4 relevés sur 13.

- le groupement 7 (15 relevés) de la station 3 possède 4 espèces: Pennisetum felicianum, Momordica charantia, Commelina benghalensis et Asystasia gangetica (Tableau 7). Ce groupe couvre une longueur de $30 \mathrm{~m}$. La moyenne d'espèces pour ce groupe est de 2. Pennisetum felicianum et Asystasia gangetica sont des espèces caractéristiques de ce groupe. Elles apparaissent dans 11 et 9 relevés sur 15, respectivement. Les autres espèces sont considérées comme espèces compagnes. Elles apparaissent au plus dans 4 relevés sur 13.

- le groupement 8 (11 relevés) de la station 3 possède 7 espèces: Setaria barbata, Struchium sparganophora, Synedrella nodiflora, Phyllanthus muelerianus, Costus afer, Ipomoea batatas et Chromolaena odorata (Tableau 7). Ce groupe couvre une longueur de $22 \mathrm{~m}$. La moyenne d'espèces pour ce groupe est de 1. Struchium sparganophora et Synedrella nodiflora sont les espèces caractéristiques de ce groupe. Elles apparaissent dans 5 relevés sur 11. Les autres espèces sont considérées comme espèces compagnes et apparaissent au plus dans 2 relevés sur 11

$\begin{aligned} & \text { Variabilité spatiale des macrophytes en } \\ & \text { fonction des paramètres physico- } \\ & \text { chimiques }\end{aligned}$
Les axes $\mathrm{F} 1(58,45 \%)$ et $\mathrm{F} 2(21,77 \%)$ du plan 1-2 de l'ACP individualisent trois groupes (Figure 6). Les groupes I et II sont constitués d'espèces exclusives et le groupe III constitué d'espèces communes à tous les groupements. Le groupe I comprend le groupement 1 provenant de la station 1 (Tableau 5; Figure 6). Il est composé de 4 espèces spécifiques (Echinochloa pyramidalis, 
Hibiscus esculentum, Ludwigia adscendens et Alstonia boonei. Elles sont positivement corrélées conductivité $(\mathrm{r}=0,68)$, à la salinité $(\mathrm{r}=0,50)$ et à la DBO5 $(\mathrm{r}=0,75)$. Le groupe II est constitué des groupements 2, 3, 4, 5, 7 et 8 provenant des stations 1,2 et 3 (Tableaux 5, 6 et 7; Figure 6) et renferme comme richesse spécifique Dioda scandens, Solanum nigrum, Cassia obtusifolia, Synedrella nodiflora, Costus afer, Commelina benghalensis, Cyperus articulatus, Eleusine indica, Panicum maxicum, Vernonia amygdalina, Leersia hexandra, Ageratum conyzoides, Acroceras amplectens,
Momordica charantia et Mimosa pudica. Elles sont corrélées positivement au phosphore $(r=0,68)$ et oxygène dissous $(r=$ $0,69)$. Le groupe III comprend le groupement 6 provenant de la station 2 et est constitué des espèces appartenant aux groupes I et II (Tableau 6; Figure 6). Les espèces communes aux groupes I, II et III sont: Ipomoea batatas, Asystasia gangetica, Phyllanthus muelerianus; Chromolaena odorata; Amaranthus viridus; Setaria barbata; Struchium sparganophora; Ipomea purpurea et Momordica charantia.

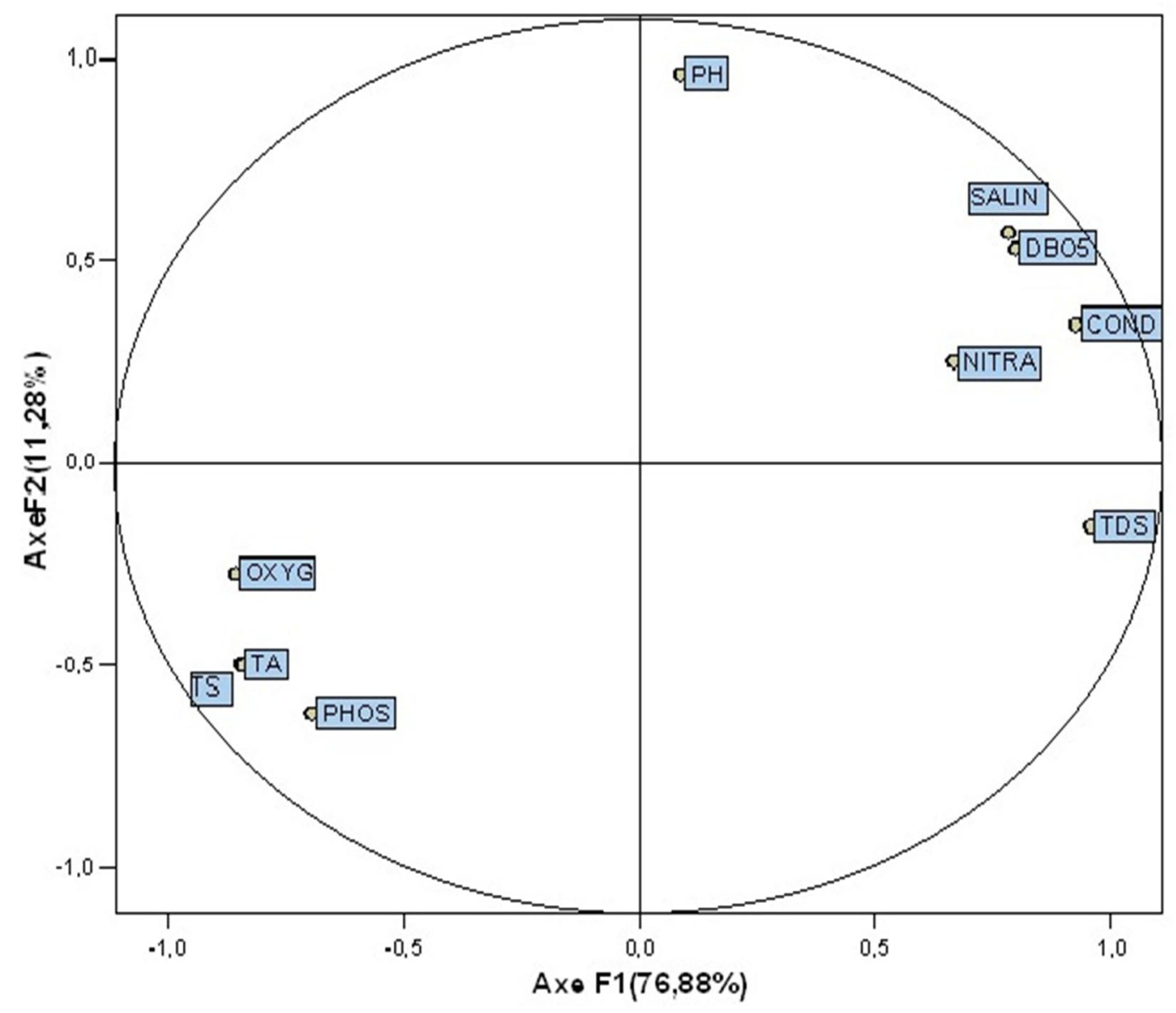

Figure 2 : Plan factoriel F1 x F2 des variables physico-chimiques. NITRA : nitrates; DBO5 (demande en oxygène) COND : conductivité électrique ; SALIN : salinité ; TDS (Solides Totaux Dissous) OXYG : oxygène; TA : température ambiante; TS température de surface ; PHOS : phosphore ; $\mathrm{pH}: \mathrm{pH}$ de l'eau. 


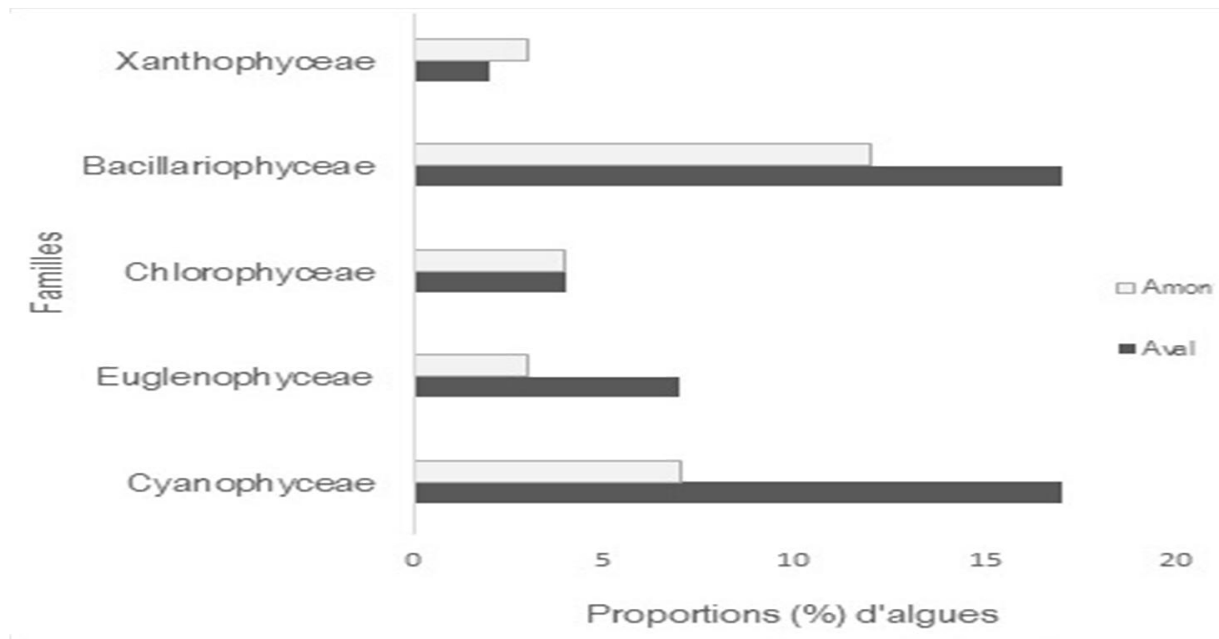

Figure 3 : Proportion d'algues (\%) en amont et en aval sur la rivière Nkam à Yabassi.

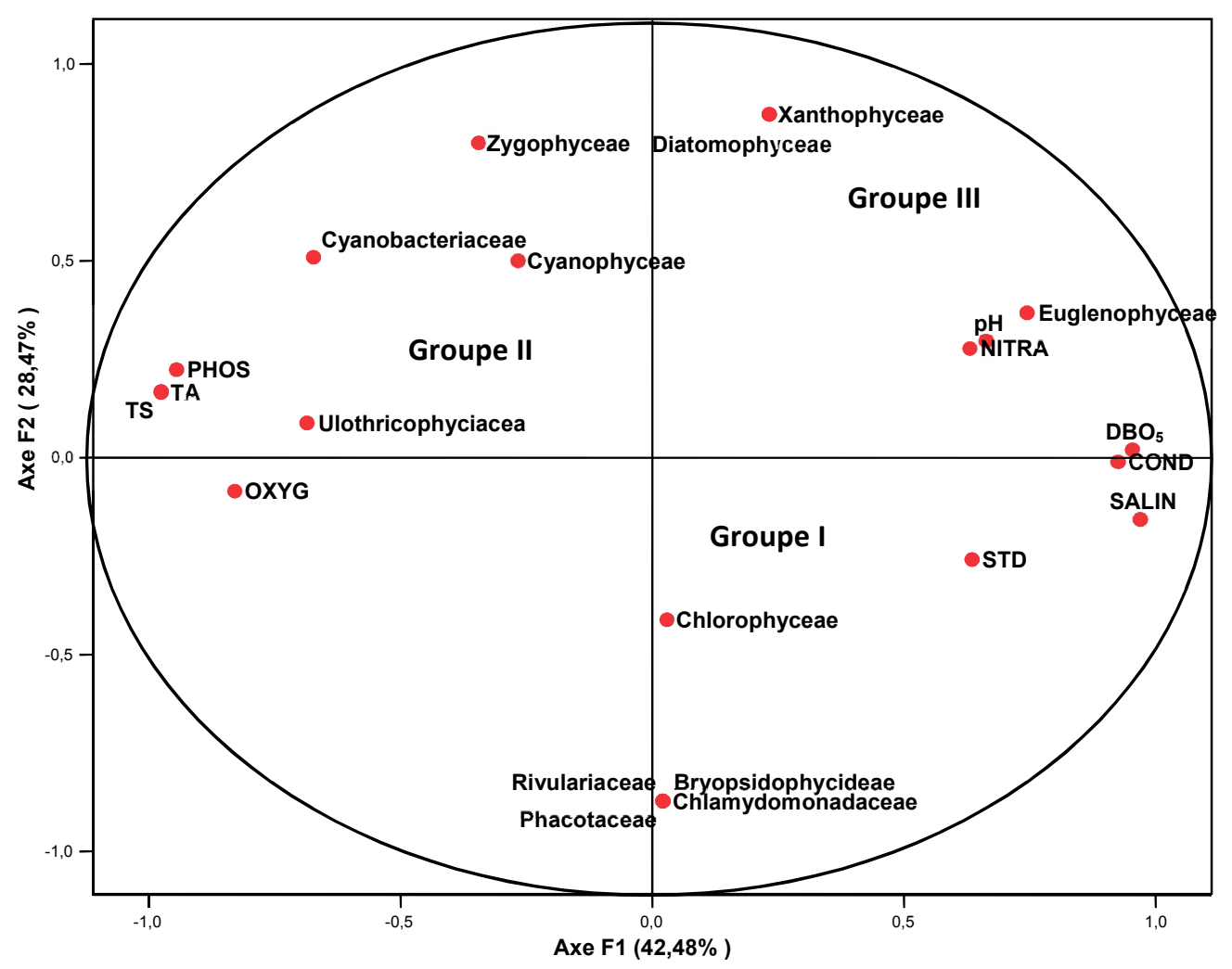

Figure 4 : Plan factoriel F1 x F2 des variables physico-chimiques en fonction du phytoplancton. NITRA : nitrates; DBO5 (demande biologique en oxygène); COND : conductivité électrique ; SALIN : salinité ; TDS (solides totaux dissous); OXYG : oxygène; TA: température ambiante; TS : température de surface ; PHOS : phosphore ; $\mathrm{pH}: \mathrm{pH}$ de l'eau. 


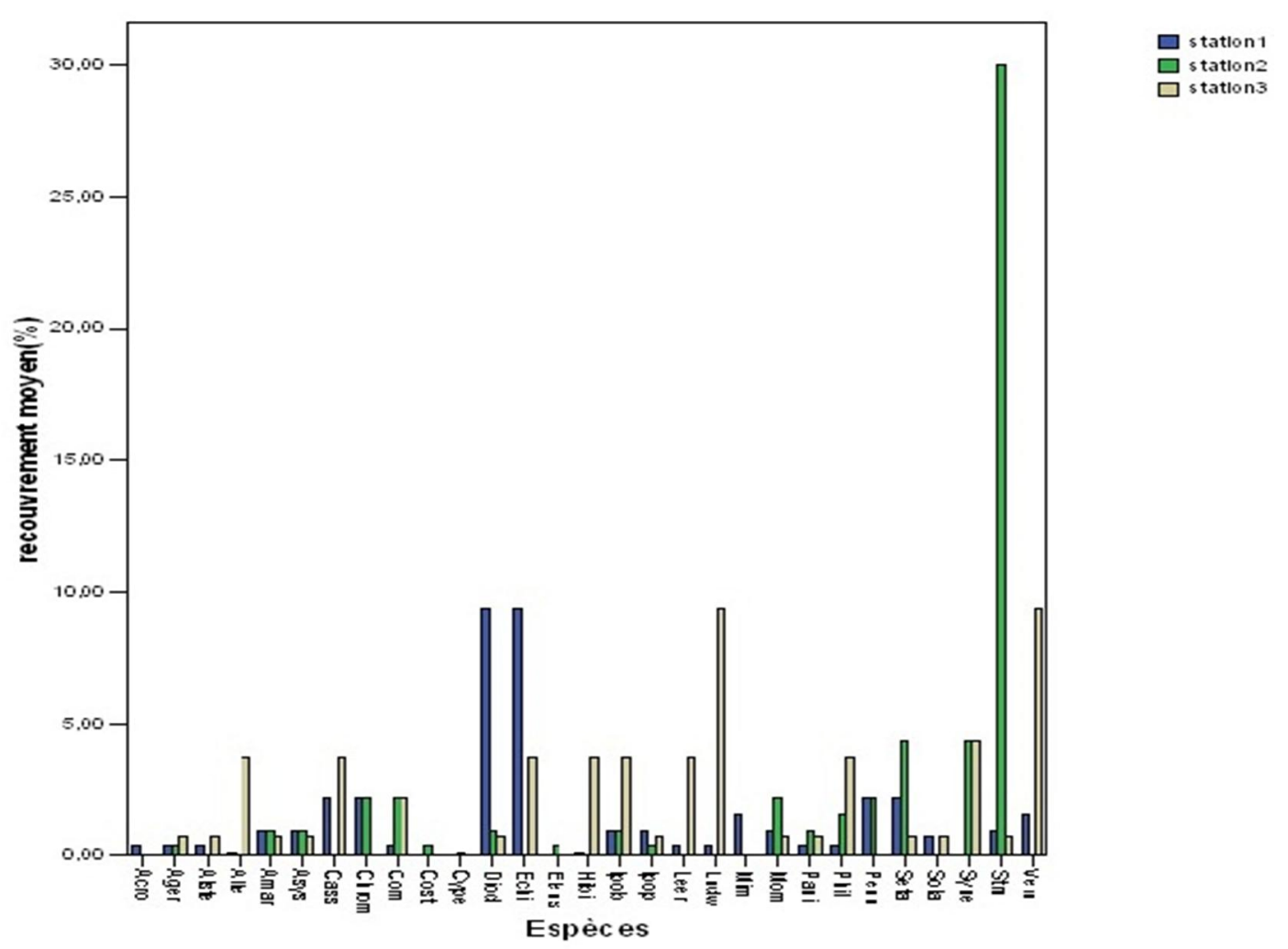

Figure 5: Recouvrement moyen (\% RM) d'espèces dans l'ensemble des stations étudiées.

Acroceras amplectens = Acro ; Ageratum conyzoides = Ager; Alstonia boonei $=$ Alste; Alternanthera $s p=$ Alte; Amaranthus viridus $=$ Amar $;$ Asystasia gangetica $=$ Asys $;$ Cassia obtusifolia $=$ Cass $;$ Chromolaena odorata $=$ Chrom ; Commelina benghalensis $=$ Com ; Costus afer $=$ Cost $;$ Cyperus articulates $=$ Cype $;$ Diodia scandens $=$ Diod; Echinochloa pyramidalis $=$ Echi; Eleusine indica $=$ Eleus; Hibiscus esculentum = Hibi; Ipomoea batatas = Ipob $;$ Ipomoea purpurea $=$ Ipop $;$ Leersia hexandra $=$ Leer $;$ Ludwigia adscendens $=\mathrm{Ludw} ;$ Mimosa pudica $=$ Mim; Momordica charantia $=$ Mom $;$ Panicum maxicum $=$ Pani; Phyllanthus muelerianus $=$ Phil $;$ Pennisetum felicianum $=$ Penn; Setaria barbata $=$ Seta; Solanum nigrum $=$ Sola; Synedrella nodiflora $=$ Syne; Struchium sparganophora $=$ Stru; Vernonia amygdalina $=$ Vern.

Tableau 1: Valeurs moyennes des paramètres physico-chimiques des stations d'étude.

\begin{tabular}{lcccccc}
\hline Paramètre & \multicolumn{3}{c}{ Station 1 (amont) } & \multicolumn{3}{c}{ Station 3 (aval) } \\
\cline { 2 - 7 } & Moy 1 & Ecart-type & Variance & Moy 2 & Ecart-type & Variance \\
\hline TA $\left({ }^{\circ} \mathrm{C}\right)$ & 29,35 & & & 30,24 & & \\
TS $\left({ }^{\circ} \mathrm{C}\right)$ & 27,47 & & & 28,65 & & \\
$\mathrm{STD}(\mathrm{ppm})$ & 185,58 & 26,95 & 726,48 & 176,44 & 0,14 & 0,02 \\
$\mathrm{COND}(\mu \mathrm{S} / \mathrm{cm})$ & 213,47 & 22,81 & 520,19 & 217,63 & 17,85 & 318,53 \\
$\mathrm{Sal}(\mathrm{ppm})$ & 166,76 & 23,72 & 562,65 & 156,11 & 31,26 & 977,26 \\
$\mathrm{pH}$ & 7,06 & 0,09 & 0,01 & 7,10 & 0,16 & 0,02 \\
$\mathrm{O}_{2}(\mathrm{mg} / \mathrm{l})$ & 4,22 & 1,18 & 1,40 & 3,54 & 0,95 & 0,91 \\
$\mathrm{NO}_{3}^{-}(\mathrm{mg} / \mathrm{l})$ & 0,46 & 0,24 & 0,06 & 0,57 & 0.03 & 0,00 \\
$\mathrm{PO}_{4}{ }^{2-}(\mathrm{mg} / \mathrm{l})$ & 0,09 & 0,01 & 0,01 & 0,17 & 0,02 & 0,04 \\
$\mathrm{DBO}_{5}(\mathrm{mg} / \mathrm{l})$ & 2,02 & 0,71 & 0,51 & 2,27 & 1,03 & 1,07 \\
\hline
\end{tabular}

Température ambiante (TA), Température de surface (TS); Solides totaux dissous (STD), Conductivité électrique (COND), Salinité (Sal.), Oxygène dissous $\left(\mathrm{O}_{2}\right)$; nitrates $\left(\mathrm{NO}_{3}^{-}\right)$, Phosphate $\left(\mathrm{PO}_{4}^{2-}\right)$; Demande Biologique en Oxygène (DBO5). 
Tableau 2: Valeurs moyennes de l'indice de diversité de Shannon Weaver (H') et d'équitabilité (E) dans les stations 1 et 3 .

\begin{tabular}{lccccc}
\hline Indice & Stations & N & Moyenne & Ecart-type & Erreur standard \\
\hline Shannon & 1 & 4 & 4,09 & 0,20 & 0,10 \\
(H') & 3 & 2 & 4,52 & 0,72 & 0,51 \\
Equitabilité (EQ) & 1 & 4 & 0,97 & 0,01 & 0.01 \\
& 3 & 2 & 1,00 & 0,13 & 0,09 \\
\hline
\end{tabular}

Tableau 3: Valeurs moyennes de l'indice de Shannon Weaver (H') et d'équitabilité de Pielou (E) des macrophytes dans les stations 1,2 et 3 .

\begin{tabular}{lccccc}
\hline Indice & Stations & N & Moyenne & Ecart-type & Erreur standard \\
& & & & & \\
\hline Shannon (H') & 1 & 2 & 3,70 & 0,08 & 0,05 \\
& 2 & 5 & 2,70 & 0,73 & 0,32 \\
Equitabilité (EQ) & 3 & 2 & 2,28 & 0,14 & 0,10 \\
& 1 & 2 & 0,88 & 0,10 & 0.09 \\
& 2 & 5 & 0,81 & 0,12 & 0,06 \\
& 3 & 2 & 0,79 & 0,11 & 0,04 \\
\hline
\end{tabular}

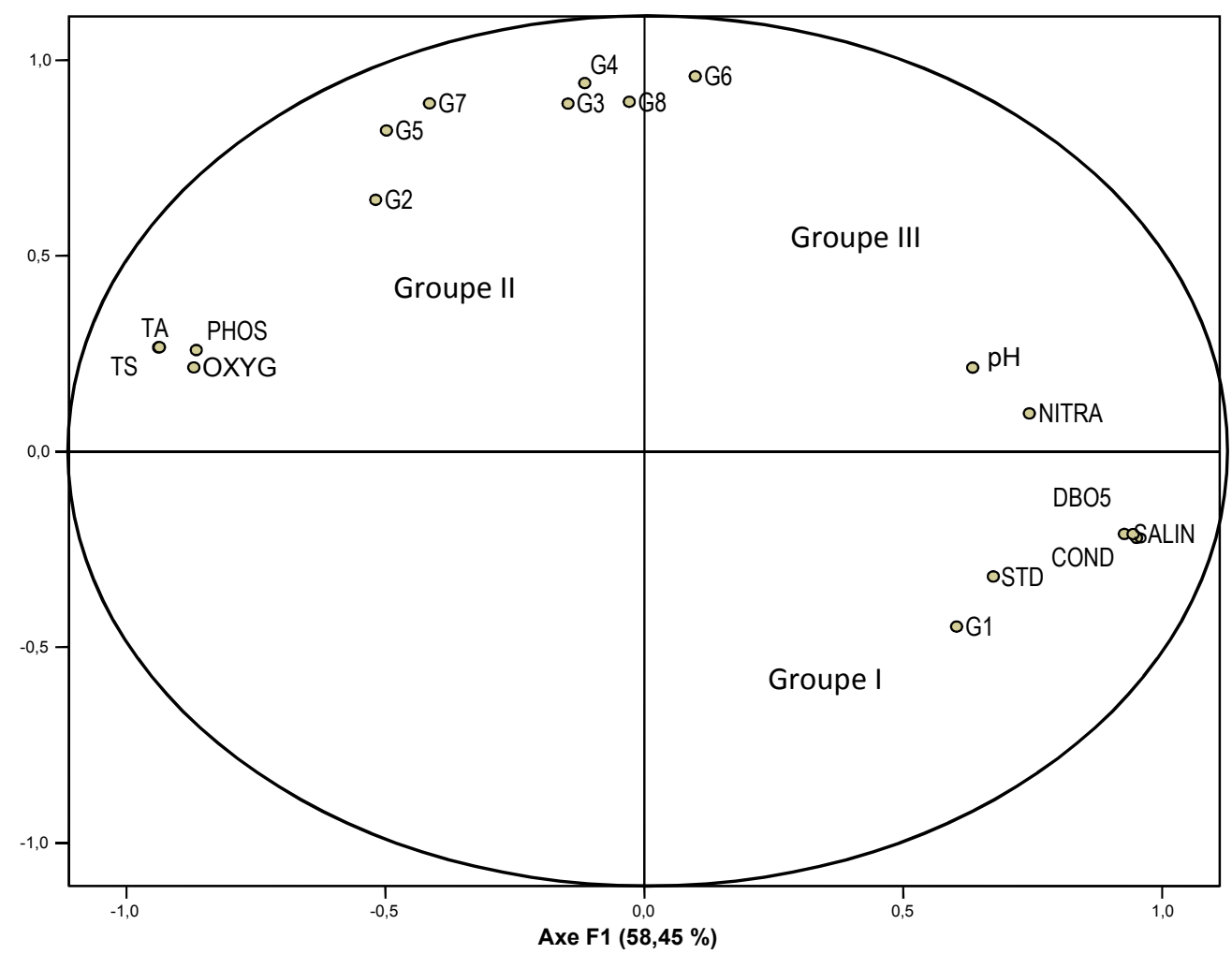

Figure 6: Plan F1 x F2 des macrophytes en fonction des paramètres physico-chimiques. G1 (groupement 1) ; G2 (groupement 2) G3 (groupement 3) ; G4 (groupement 4) ; G5 (groupement 5) ; G6 (groupement 6) ; G7 (groupement 7); G8 (groupement 8); Température atmosphérique $\left({ }^{\circ} \mathrm{C}\right)(\mathrm{TA})$, Température de surface $(\mathrm{TS})\left({ }^{\circ} \mathrm{C}\right)$; solides totaux dissous ( $\mathrm{ppm})(\mathrm{STD})$, Conductivité électrique $(\mu \mathrm{S} / \mathrm{cm})(\mathrm{COND})$, Salinité (ppm), Oxygène dissous $(\mathrm{mg} / \mathrm{l})(\mathrm{OXYG})$; Nitrates (mg/l) (NITRA) ; Phosphore (mg/l) (PHOS); Demande biologique en oxygène $(\mathrm{mg} / \mathrm{l})\left(\mathrm{DBO}_{5}\right)$. 
Tableau 4: Caractéristiques phyto-sociologiques des stations d'étude.

\begin{tabular}{lccccccc}
\hline Stations & Alt $\mathbf{( m )}$ & $\mathbf{P t}(\mathbf{\%})$ & $\mathbf{L t} \mathbf{( m )}$ & $\left.\mathbf{S . E} \mathbf{( m}^{\mathbf{2}}\right)$ & $\mathbf{L m}$ & Omb. & M.E. \\
\hline 1 & $-1,00$ & 5,00 & 31,00 & 62,00 & 100,00 & 50,00 & habité, cultures \\
\hline 2 & 16,00 & 0,50 & 110,00 & 220,00 & 100,00 & 0,25 & habité, cultures \\
\hline 3 & 1,00 & 0,50 & 55,00 & 110,00 & 50,00 & 0,25 & habité, cultures
\end{tabular}

Alt: altitude; Pt : pente; S.E. : surface échantillonnée; Lm. : largeur du lit mouillée; Omb. : ombrage des berges ; M.E : milieux environnants; $\mathrm{Lt}$ : longueur totale.

Tableau 5: Groupement 1 à Echinochloa pyramidalis; groupement 2 à Diodia scandens ; groupement 3 à Pennisetum felicianum et groupement 4 à Ipomoea purpurea.

\begin{tabular}{|c|c|c|c|c|}
\hline Numéro de quadrats & 1 & 4 & 3 & 2 \\
\hline Recouvr ement total (\%) & 100 & 100 & 100 & 99 \\
\hline Nombre d'espèces & 15 & 14 & 12 & 9 \\
\hline \multicolumn{5}{|l|}{ Surface recouverte $\left(62 \mathrm{~m}^{2}\right)$} \\
\hline \multicolumn{5}{|l|}{ Echinochloa Pyramidalis } \\
\hline Alstonia boonei & & & 1 & 1 \\
\hline Ludwigia adscendens palustris & 1 & 1 & 1 & 1 \\
\hline Phyllanthus muelerianus & 1 & & 4 & 4 \\
\hline Ipomoea batatas & & 2 & 3 & 1 \\
\hline Asystasia gangetica & 1 & 1 & 2 & 1 \\
\hline Hibiscus esculentum & 1 & & 1 & 1 \\
\hline Echinochloa Pyramidalis & & 2 & & 1 \\
\hline Chromolaena odorata & 2 & & & 1 \\
\hline Diodia scandens & 2 & 1 & 2 & \\
\hline Solanum nigrum & 1 & 1 & 1 & \\
\hline Panicum maxicum & & 1 & 1 & \\
\hline Amaranthus viridus & 1 & 1 & 1 & \\
\hline Cassia obtusifolia & 1 & & 1 & \\
\hline Alternanthera sp & & & 1 & \\
\hline Pennisetum felicianum & 1 & 3 & & \\
\hline Vernonia amygdalina & 3 & 1 & & \\
\hline Setaria barbata & 1 & 1 & & \\
\hline Leersia hexandra & & 1 & & \\
\hline Ipomoea purpurea & & 1 & & \\
\hline Ageratum conyzoides & 2 & 1 & & \\
\hline Struchium sparganophora & 1 & & & \\
\hline
\end{tabular}


V. D. TAFFOUO et al. / Int. J. Biol. Chem. Sci. 11(4): 1766-1784, 2017

Tableau 6: Groupement 5 à Pennisetum felicianum et groupement 6 à Setaria barbata.

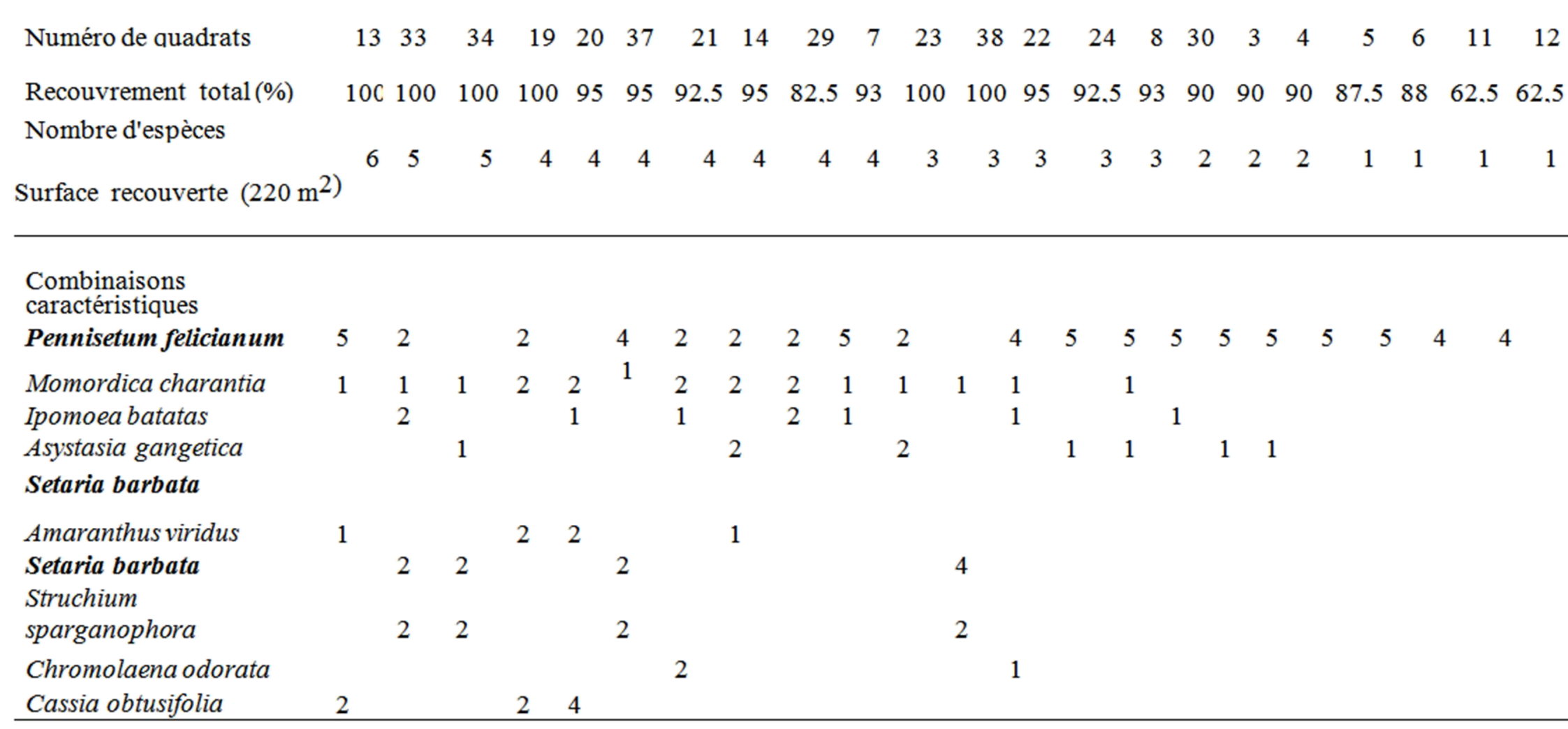

L'échelle de Braun-Blanquet : + =recouvrement $<1 \% ; 1=1$ à $5 \% ; 2=5$ à $25 \% ; 3=25$ à $50 \% ; 4=$ recouvrement 50 à $75 \% ; 5=$ recouvrement 75 à $100 \%$. Fc $=$ classe des fréquences. 
Tableau 7: Groupement 7 à Panicum maximum et groupement 8 à Struchium sparganophora .

\begin{tabular}{|c|c|c|c|c|c|c|c|c|c|c|c|c|c|c|c|c|c|c|}
\hline Numéro de quadrats & 13 & 1 & 8 & 4 & 5 & 20 & 3 & 6 & 7 & 14 & 19 & 2 & 17 & 16 & 12 & 18 & 11 & 15 \\
\hline Recouvrement total (\%) & 100 & 100 & 100 & 100 & 100 & 98,5 & 93,5 & 100 & 100 & 100 & 100 & 100 & 100 & 100 & 100 & 100 & 100 & 88 \\
\hline Nombre d'espèces & 7 & 6 & 6 & 5 & 5 & 5 & 4 & 4 & 4 & 4 & 4 & 3 & 3 & 2 & 2 & 2 & 2 & 1 \\
\hline \multicolumn{19}{|l|}{ Surface recouverte $\left(110 \mathrm{~m}^{2}\right)$} \\
\hline \multicolumn{19}{|l|}{$\begin{array}{l}\text { Combinaisons } \\
\text { caractéristiques }\end{array}$} \\
\hline Panicum maxicum & 5 & 1 & 1 & 4 & 4 & 4 & 5 & 4 & 2 & 3 & 4 & 4 & & 2 & & & & \\
\hline Asystasia gangetica & & & 1 & 2 & 1 & & 2 & 2 & 2 & & & 3 & 1 & & & & 3 & \\
\hline Momordica charantia & & & 4 & & & & & 3 & 4 & & 1 & & 1 & & 3 & & & \\
\hline Commelina benghalensis & & & 2 & 3 & & 1 & 1 & & & & & & & & & 2 & & \\
\hline Struchium sparganophora & & & & & & & & & & 4 & & & & & & & & \\
\hline Setaria barbata & & & & & 1 & 2 & & & & 3 & & & & & 3 & & 2 & \\
\hline Synedrella nodiflora & & & & & & 2 & & & & & 2 & & & 5 & & 4 & & 2 \\
\hline Phyllanthus muelerianus & & & & & & & & & & & & & 1 & & & & & \\
\hline Costus afer & & & & & & & & & 1 & & & & & & & & & \\
\hline Ipomoea batatas & & & & & & 1 & & & & & 1 & & & & & & & \\
\hline Chromolaena odorata & & & & & 2 & & & & & & & & & & & & & \\
\hline
\end{tabular}

L'échelle de Braun-Blanquet : $+=$ recouvrement $<1 \% ; 1=1$ à $5 \% ; 2=5$ à $25 \% ; 3=25$ à $50 \% ; 4=$ recouvrement 50 à $75 \% ; 5=$ recouvrement 75 à $100 \%$.FC $=$ classe des fréquences.

\section{DISCUSSION}

\section{Paramètres physico-chimiques des stations d'étude}

La conductivité électrique est un indice de l'abondance des ions dans l'eau. Elle permet d'estimer le degré de minéralisation et présente souvent un gradient croissant de l'amont vers l'aval (Berryman, 2006). Dans la présente étude, elle fluctue entre $213 \mu \mathrm{S} / \mathrm{cm}$ en amont et 217 $\mu \mathrm{S} / \mathrm{cm}$ en aval (Tableau 1). La valeur moyenne $(215 \mu \mathrm{S} / \mathrm{cm})$ reste faible par rapport au seuil fixé à $1500 \mu \mathrm{S} / \mathrm{cm}$ (Barnabe and Barnabe-Quet, 2000), indiquant une minéralisation attribuée aux eaux des affluents avoisinants et aux activités de carrière exercées en amont (Dibong et Ndjouondo, 2014). D'après Barnabe and Barnabe-Quet (2000), une conductivité électrique supérieure à $1500 \mu \mathrm{S} / \mathrm{cm}$ fait considérer une eau comme inutilisable dans les zones irriguées car ayant un impact sur le développement des organismes et sur les équilibres chimiques, suivant l'affinité de chaque organisme avec la concentration des ions. Par rapport à la valeur moyenne de conductivité électrique, l'eau de la rivière
Nkam serait propice à l'arrosage des cultures maraîchères qui sont le plus souvent pratiquées le long du cours d'eau. La valeur moyenne du $\mathrm{pH}(7,08)$ dans les stations 1 et 3 indique une neutralité des eaux pendant la période de l'étude; la valeur observée serait liée à la forte présence des macrophytes fixés. En effet, au niveau des stations 1 et 3, l'acide humique qui provient de la décomposition des macrophytes morts contribuerait à baisser le pH (Atanle, 2012). Hade (2002) a rapporté que lorsque le $\mathrm{pH}$ est élevé, l'azote ammoniacal $\left(\mathrm{NH}^{+}\right)$devient toxique tandis que pour un $\mathrm{pH}$ inférieur à 8 , l'azote ammoniacal a peu d'influence sur la faune et la flore. Les nitrates $\left(\mathrm{NO}_{3}{ }^{-}\right)$ constituent une source d'azote très importante pour la croissance des algues car ils sont fonction $\mathrm{du} \mathrm{pH}$ (Wetzel et Likens, 2000). La valeur moyenne de $\mathrm{NO}_{3}{ }^{-}$en aval $(0,57 \mathrm{mg} / \mathrm{l})$ est supérieure au seuil fixé à 0,45 $\mathrm{mg} / \mathrm{l}$ (Tableau 1), ce résultat indique que les eaux étudiées sont assujetties à un risque de pollution par $\mathrm{NO}_{3}^{-}$(Makhoukh, 2011). Pour le phosphate $\left(\mathrm{PO}_{4}{ }^{2-}\right)$, Priso (2012) a trouvé dans la rivière Kondi, les valeurs de 0,664 $\mathrm{mg} / \mathrm{l}$ en amont et de $0,166 \mathrm{mg} / \mathrm{l}$ en aval. La 
valeur moyenne trouvée en aval de Kondi $(0,166 \mathrm{mg} / \mathrm{l})$ est comparable à celle observée en aval du Nkam (0,17 mg/l) (Tableau 1). Le seuil de l'eutrophisation étant fixé à 0,03 $\mathrm{mg} / \mathrm{l}$ de $\mathrm{PO}_{4}{ }^{2-}$ (Hade, 2002). Le taux de $\mathrm{PO}_{4}{ }^{2-}$ dissous en amont $(0,09 \mathrm{mg} / \mathrm{l})$ est supérieur au seuil de $0,03 \mathrm{mg} / 1$, ce qui indiquerait un début d'eutrophisation de la rivière en amont. L'essentiel de $\mathrm{PO}_{4}{ }^{2-}$ mesuré dans la rivière Nkam aurait une origine exogène. En effet, la pratique de l'agriculture avec utilisation des engrais chimiques (vergers, cultures maraichères et vivrières) le long de la rivière Nkam devrait constituer une source importante de phosphate transporté vers le fleuve par les eaux de ruissellement. Le $\mathrm{PO}_{4}^{2-}$ proviendrait aussi de la décomposition des déchets en amont et le long de la rivière Nkam (Atanle, 2012). D'après les travaux de Sane (2006) au Sénégal, l'absorption du phosphore dans le sédiment et les apports des rivières tributaires devraient constituer une source importante de phosphate transporté vers les fleuves par les eaux de ruissellement. L'analyse de l'organisation des variables physico-chimiques sur les plans factoriels F1 x F2 de l'ACP a révélé que l'axe factoriel $\mathrm{F} 1$ est défini par la matière organique et les sels nutritifs (salinité, $\mathrm{NO}_{3}{ }^{-}$) (Figure 2). $\mathrm{NO}_{3}^{-}$proviendrait des engrais utilisés en agriculture en amont et autour la rivière Nkam. Atante et al. (2012) a fait la même observation dans les eaux du lac de Zowla en Côte d'Ivoire. Selon ces auteurs, les orthophosphates et les nitrates auraient une même origine et proviendraient des engrais utilisés en agriculture en amont et autour du lac de Zowla.

\section{Corrélation entre les variables physico- chimiques et phytoplancton}

Les corrélations entre les variables environnementales et les variables biologiques révèlent des fortes corrélations positives d'après la matrice de corrélation de Pearson entre la densité des Chlorophyceae, Bryopsidophycideae Chlamydomonadaeae, Phacotaceae, Rivulariaceae et la turbidité (Figure 4). Cette turbidité élevée serait due à l'abondance de la matière organique particulaire en suspension dans l'eau. Benabdellouahad (2006) et Atanle et al. (2012) ont fait les mêmes constatations sur les Diatomophyceae dans l'estuaire du Bou Regreg au Maroc et dans le lac Zowla au Togo. Selon ces chercheurs, certaines espèces foisonnent dans les eaux beaucoup plus turbides et avec des grands taux de nutriments.

\section{Diversité du phytoplancton}

Les résultats de la présente étude montrent que chez le phytoplancton l'indice de diversité de Shannon-Weaver (4,09 à 4,54 bits) et d'équitabilité de Pielou (0,97 à 1) dans les stations 1 (amont) et 3 (aval) respectivement, sont élevés dans les deux sites (Tableau 2). La comparaison par la méthode de Ireland (2010) montre qu'il n'ya pas de différence significative entre les indices de diversité issus de la station 1 et 3 . La régularité supérieure à 0,5 en amont et aval de la rivière Nkam montre qu'un maximum d'espèces participe au recouvrement de la surface de l'eau (Priso et al., 2012). La régularité tend vers 1 , ce qui suggère que toutes les espèces ont la même abondance (Tableau 2).

\section{Diversité des macrophytes}

Chez les macrophytes les valeurs d'indice de diversité de Shannon Weaver $(2,28$ à 3,70 bits $)$ traduisent une grande diversité spécifique en amont (Tableau 3). Cependant l'équitabilité de Piélou ( 0,79 à $0,88)$ témoigne une bonne répartition des individus au sein des espèces (Tiokeng et al., 2015). Ces résultats corroborent ceux de Priso et al. (2012), Dibong et Ndjouondo (2014) qui ont observé le même gradient de distribution des macrophytes dans la rivière Kambo et Kondi. En effet, la structuration des macrophytes entre l'amont et l'aval sur la rivière Kondi serait due à la présence de certains éléments mais peut également être due aux crues qui laissent les dégâts sur la végétation (Priso et al., 2012). D'après Dibong et al. (2014), cette perturbation liée à la distribution des macrophytes le long du cours 
d'eau serait marquée par les activités observées sur le terrain. Barendregt et Bio (2003) pensent que la lumière, la température, les vitesses mais aussi le phosphore, l'azote et le carbone inorganique sont des facteurs qui influencent la croissance macrophytique d'un cours d'eau.

\section{Groupements végétaux en relation avec le milieu}

Au total 70 relevés de $1 \mathrm{~m}^{2}$ ont été réalisés. La végétation du site étudié comporte des groupements végétaux dans lesquels cohabitent des espèces qui y trouvent des conditions favorables (Tableaux 5, 6 et 7). Par rapport aux relevés, les résultats de cette présente étude différent de ceux obtenus par Priso e t a 1. (2012), Dibong et Ndjouondo (2014) excepté la richesse spécifique des macrophytes dominée par les Poaceae et les Asteraceae. Au total quatre-vingt-dix-sept espèces de macrophytes ont été recensées dans la rivière Kondi contre 32 espèces répertoriées le long de la rivière Nkam. Ces différences observées pourraient s'expliquer par le fait que les groupements végétaux peuvent être répartis en fonction du stade d'évolution de la végétation. De même, les macrophytes ne se répartissent pas au hasard dans un milieu et présentent une zonation longitudinale selon les cours d'eau (Priso et al., 2012). Ainsi, les facteurs physico-chimiques ne sont pas les seuls responsables de la répartition des principaux groupements (Barnabe and Barnabe-Quet, 2000; Ngueguim et al., 2010).

\section{Conclusion}

Cette étude montre que la richesse spécifique du phytoplancton et des macrophytes dans la rivière Nkam est diversifiée : 80 espèces phytoplanctoniques distribuées dans 14 familles ont été répertoriées tandis que chez les macrophytes, 32 espèces réparties dans 19 familles ont été recensées. De l'amont vers l'aval, les groupements végétaux se répartissent suivant un gradient de minéralisation amont-aval. Les éléments tels que la conductivité électrique, la salinité, la demande biochimique en oxygène, le phosphate et l'oxygène dissous influent sur la répartition des macrophytes au niveau de la rivière Nkam. Les valeurs d'indice de diversité de Shannon Weaver traduisent une grande diversité spécifique du phytoplancton (4,09 à 4,54 bits) et des macrophytes (2,28 à 3,70 bits) de la rivière Nkam. L'équitabilité de Piélou qui varie de 0,96 à 1 pour le phytoplancton et de 0,52 à 0,96 pour les macrophytes témoigne une bonne répartition des individus au sein des espèces. Les Phacotaceae, Rivulariaceae, Bryopsidophycideae, Chlamydomonadaceae et Chlorophyceae sont indicatrices d'une intense minéralisation $\mathrm{du}$ milieu. Les Diatomophyceae, Euglenophyceae et Xanthophyceae présentent une grande plasticité écologique.

\section{REMERCIEMENTS}

Les auteurs remercient Ndjouondo Gildas Parfait, Siyou Jacques et Hervé Gimbis Millo pour leur assistance sur le terrain et au laboratoire.

\section{CONFLIT D'INTERETS}

Aucun conflit d'intérêt n'est à signaler.

\section{CONTRIBUTIONS DES AUTEURS}

VDT et RAIS ont collecté les données sur le terrain. RAIS a effectué les analyses au laboratoire, VDT, LOM et MTE ont participé à la rédaction de cet article.

\section{REFERENCES}

Atanle K, Moctar L, Bawa, Kokou K, Gbandi DB. 2012. La caractérisation physicochimique et diversité phytoplanctonique des eaux du lac de Zowla (Lac Boko), au Togo. J. Appl. BioSci., 64: 48474857.

Barnabe G, Barnabe-Quet R. 2000. Ecology and Management of Coastal Waters: The Aquatic Environment. Springer: UK.

Barendregt A, Bio AMF. 2003. Relevant variables to predict macrophyte communities in running waters. Ecological Modelling, 160(3): 205-217. 
https://doi.org/10.1016/S03043800(02)00254-5

Bellinger EG, Sigee DC. 2015. Freshwater Algae: Identification, Enumeration and Use as Bioindicators (2nd edn). Wiley Blackwell: USA.

Benabdellouahad S. 2006. Structure, dynamique et typologies physicochimiques et phytoplanctoniques de l'estuaire du Bou Regreg (côte atlantique marocaine). Thèse de Doctorat, Université Mohammed VAgdal de Rabat, Maroc, p. 252.

Berho C. 2008. Procédures d'échantillonnage des eaux souterraines en vue d'une analyse microbiologie. Etat de l'Art. Rapport final BRGM/RP-56405-FR, p.26.

Berryman D. 2006. Établir des critères de qualité de l'eau et des valeurs de référence pour le phosphore, selon les éco-régions: opportunité, faisabilité et premier exercice exploratoire. Direction du suivi de l'état de l'environnement, Ministère du Développement durable, de l'Environnement et des Parcs.

Braun-Blanquet J. 1964. Pflanzensoziologie. SpringerVerlag: Wien.

Buchez A, Dorigo U, Rimet F. 2010. Surveillance des impacts environnementaux d'effluents aqueux de sites industriels par les diatomées dulçaquicoles. Etude Record $\mathrm{n}^{\circ} 07-$ 1016/1A, p. 175.

Diagne I, Drame S, Ndiaye M, Nddiaye B, Diop A. 2017. Caractérisation physicochimique et contamination métallique des eaux usées déversées au niveau de la baie de Hann (Dakar/Sénégal). Int. J. Biol. Chem. Sci., 11(1): 462-472. DOI: http://dx.doi.org/10.4314/ijbcs.v11i1.37

Dibong SD, Ndjouondo GP. 2014. Inventaire floristique et écologie des macrophytes aquatiques de la rivière Kambo à Douala (Cameroun). J. Appl. BioSci., 80: 7147-7160. http://dx.doi.org/ 10.4314/jab.v80i1.15

Evangelista V, Barsanti L, Frassanito AM, Passareli V, Gualtieri P. 2007. Algal
Toxins, Nature, Occurrence, Effect and Detection. Springer: Netherlands.

Hade A. 2007. Nos Lacs: les Connaître pour Mieux les Protéger. Edition FIDES: Quebec.

Ireland CR. 2010. Experimental Statistics for Agriculture and Horticulture. Cambridge University Press, Cambridge.

Kemka N, Njine T, Zébazé SH, Togouet D, Niyitegeka MN, Monkiedje J, Demannou S, Foto M. 2004. Phytoplancton du lac municipal de Yaoundé (Cameroun): Succession écologique et structure des peuplements. Revue des Sciences de l'Eau, 17: 301316.

Kpidi YH, Yapo OB, Ballet TG, Ohou-Yao MJ. 2017. Variabilité journalière de la qualité physico-chimique du lac M'koa de Jacqueville (Côte d'Ivoire). Int. J. Biol. Chem. Sci., 11(2): 901-910. DOI: https://dx.doi.org/10.4314/ijbcs.v11i2.29

Makhoukh M, Sbaa M, Berthoud A, Clooster VM. 2011. Contribution à l'étude physico-chimique des eaux superficielles de l'oued moulouya (Maroc oriental). Larhyss J., 9: 149-169.

Masens Dy B. 1997. Etude phytosociologique de la région de Kikwit (Bandudu, République Démocratique du Congo). Thèse Université libre de Bruxelles, Belgique, p. 398.

MINADER, 2011. Rapport annuel des activités. MINADER, p. 43.

Ngueguim JR, Zapfack L, Youmbi E, Riera B, Onana J, Foahom B, Makombu JG. 2010. Diversité floristique sous canopée en plantation forestière de MangombeEdéa (Cameroun). Biotechnol., Agron. Soc. Environ., 14: 167-176.

Niamien-Ebrottié JE, Konan KF, Edia OEA, Gourène G. 2013. Composition et variation spatio- saisonnière $\mathrm{du}$ peuplement algal des rivières côtières du Sud-Est de la Côte d'Ivoire. J. Appl. BioSc., 66: 5147-5161. 
Noukeu NA, Gouado I, Priso RJ, Ndongo D, Taffouo VD, Dibong SD, Ekodeck GE. 2016. Characterization of effluent from food processing industries and stillage treatment trial with Eichhornia crassipes (Mart.) and Panicum maximum (Jacq.). Water Resources and Industry, 16: 1-18. https://doi.org/10.1016/j.wri.2016.07.001

Priso RJ, Dibong SD, Tchinda-Metagne C, Taffouo VD, Ndongo D, Amougou A. 2010. Impacts des eaux polluées sur la croissance, les teneurs en chlorophylles et substances organiques dans les feuilles de deux Poaceae. Int. J. Biol. Chem. Sci., 4: 1122-1129.

Priso RJ, Oum GO, Ndongo D. 2012. Utilisation des macrophytes comme descripteurs de la qualité des eaux de la rivière Kondi dans la ville de Douala (Cameroun-Afrique Centrale). J. Appl. BioSci., 53: 3797-38911.

Ramade F. 2008. Proposal of ecotoxicological criteria for the assessment of the impact of pollution on environmental quality. Toxicol. Environ. Chem., 13: 189-203. http://dx.doi.org/10.1080/02772248709 357181

Reichwaldt ES, Ghadoua A. 2012. Effects of rainfall patterns on toxic cyanobacterial bloomsin a changing climate: Between simplistic scenarios and complex dynamics. Water Research, 46: 13721393. doi:10.1016/j.watres.2011.11.052

Rodier J, Legube B, Merlet N. 2009. L'Analyse de l'Eau (9è edn). Dunod: Paris.

Roxane T, Reinhard P. 2015. Caractéristiques limnologiques de 56 lacs du Québec méridional en lien avec l'état trophique. Revue des Sciences de l'Eau, 28(2): 139-162.

Sane S. 2006. Contrôle environnemental de la production primaire du lac de Guiers au Nord du Sénégal. Thèse de Doctorat, Université Cheikh Anta Diop de Dakar, Sénégal, p. 187.

Sauberer N, Zulka KP, Abensperg-Traun M, Berg HM, Bieringer G, Milasowsky N, Moser D, Plutzar C, Pollheimer M, Storch C, Trostl R, Zechmeister H, Grabherr G. 2004. Surrogate taxa for biodiversity in agricultural landscapes of Eastern Austria. Biological Conservation, 117: 181-190. doi:10.1016/S0006-3207(03)00291-X

Tiokeng B, Mapongmetsem P-M, Nguetsop VF, Tacham WN. 2015. Biodiversité floristique et régénération naturelle sur les Hautes Terre de Lebialem (Ouest Cameroun). Int. J. Biol. Chem. Sci., 9(1): 56-68. DOI: http://dx.doi.org/10. 4314/ijbcs.v9i1.6

Wetzel RG, Likens GE. 2000. Limnological Analyses ( $3^{\text {rd }}$ edn). Springer-Verlag Inc: New-York. 